\title{
Numerical Modeling of Two-Phase Hydromagnetic Flow and Heat Transfer in a Particle-Suspension through a non-Darcian Porous Channel
}

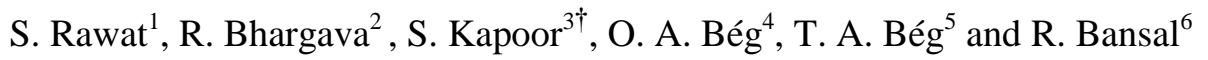 \\ ${ }^{I}$ Assistant Professor Department of Mathematics, Jubail University College (Male Branch)Jubail Industrial \\ City 31961Kingdom of Saudi Arabia \\ ${ }^{2}$ Professor, Department of Mathematics, Indian Institute of Technology, Roorkee - 247667, India. \\ ${ }^{3}$ Assistant Professor Department of Education in Science and Mathematics, Regional Institute of Education, \\ Bhubneswar (NCERT), India \\ ${ }^{4,5}$ Professor, Department of Engineering and Mathematics, Sheaf Building, Sheaf Street, Sheffield Hallam \\ University, Sheffield, S11WB, England \\ ${ }^{6}$ Reseacrh Scholar, IFTM University, Moradabad, India \\ $\dagger$ Corresponding Author Email: saurabh09.iitr@gmail.com
}

(Received December 29, 2012; accepted May 16, 2013)

\begin{abstract}
A mathematical model is presented for the steady, two-dimensional magneto-convection heat transfer of a two-phase, electrically-conducting, particle-suspension in a channel containing a non-Darcian porous medium intercalated between two parallel plates, in the presence of a transverse magnetic field. The channel walls are assumed to be isothermal but at different temperatures. The governing equations for the one-dimensional steady flow are formulated following Marble (1970) and extended to include the influence of Darcian porous drag, Forcheimmer quadratic drag, buoyancy effects, Lorentz body force (hydromagnetic retardation force) and particle-phase viscous stresses. Special boundary conditions for the particle-phase wall conditions are implemented. The governing coupled, non-linear differential equations are reduced from an (x,y) coordinate system to a one-dimensional (y) coordinate system. A series of transformations is then employed to nondimensionalize the model in terms of a single independent variable, $\eta$, yielding a quartet of coupled ordinary differential equations which are solved numerically using the finite element method, under appropriate transformed boundary conditions. The influence of for example Grashof free convection number $(\mathrm{Gr})$, Hartmann hydromagnetic number $(\mathrm{Ha})$, inverse Stokes number $\left(\mathrm{Sk}_{\mathrm{m}}\right)$, Darcy number $(\mathrm{Da})$, Forcheimmer number (Fs),particle loading parameter $\left(\mathrm{P}_{\mathrm{L}}\right)$, buoyancy parameter $(\mathrm{B})$ on the fluid-phase velocity and particle-phase velocity are presented graphically. A number of special cases of the transformed model are also studied. The mathematical model finds applications in solar collector devices, electronic fabrication, jet nozzle flows, industrial materials processing transport phenomena, MHD energy generator systems etc.
\end{abstract}

Keywords: Two-phase hydrodynamic, Particle suspension, Buoyancy, Heat transfer, Magnetic fields, Non-Darcy porous medium, Stokes number, Finite element solutions.

\section{NOMENCLATURE}

Dimensional

$\rho \quad$ density of fluid phase

t time

$\nabla \quad$ Del operator

V fluid phase velocity vector

$\mathrm{P} \quad$ hydrodynamic pressure

$\mu \quad$ dynamic viscosity of fluid phase

$\mathrm{pp} \quad$ density of particle phase

$\mathrm{N}$ interphase momentum transfer coefficient

\begin{tabular}{|c|c|}
\hline $\mathrm{Vp}$ & particle phase velocity vector \\
\hline $\mathrm{g}$ & gravitational acceleration \\
\hline B & magnetic field vector \\
\hline K & permeability of porous medium \\
\hline $\mathrm{b}$ & $\begin{array}{l}\text { Forcheimmer inertial resistance } \\
\text { parameter }\end{array}$ \\
\hline $\mathrm{c}$ & $\begin{array}{l}\text { specific heat of fluid phase at constant } \\
\text { pressure (isobaric) }\end{array}$ \\
\hline & temperature of fluid phase \\
\hline & thermal conductivity of fluid phase \\
\hline
\end{tabular}




\begin{tabular}{|c|c|}
\hline $\mathrm{cp}$ & $\begin{array}{l}\text { specific heat of particle phase at } \\
\text { constant pressure (isobaric) }\end{array}$ \\
\hline NT & interphase heat transfer coefficient \\
\hline $\mathrm{Tp}$ & temperature of particle phase \\
\hline$\mu \mathrm{p}$ & dynamic viscosity of particle phase \\
\hline $\mathrm{x}$ & direction parallel to channel \\
\hline $\mathrm{y}$ & direction transverse to channel \\
\hline $\mathrm{u}$ & velocity of fluid phase \\
\hline up & velocity of particle phase \\
\hline$\beta^{*}$ & coefficient of volumetric expansion \\
\hline s & separation of plates comprising channel \\
\hline$\omega$ & particle phase wall-slip coefficient \\
\hline \multicolumn{2}{|c|}{ Non-dimensional Parameters } \\
\hline$\eta$ & transformed y coordinate \\
\hline $\mathrm{U}$ & $\begin{array}{l}\text { dimensionless } \eta \text {-direction fluid phase } \\
\text { velocity }\end{array}$ \\
\hline Up & $\begin{array}{l}\text { dimensionless } \eta \text {-direction particle } \\
\text { phase velocity }\end{array}$ \\
\hline$\Phi$ & dimensionless temperature of fluid \\
\hline
\end{tabular}

\section{INTRODUCTION}

Hydromagnetic convection flows constitute a major branch of modern heat transfer research. Applications abound in many areas of technology including fusion processes in electrical furnaces, plasma generators, ferrohydrodynamic processing, plasma aerodynamics etc. Many single phase numerical studies of such flows have been communicated in the literature. Singh and Cowling et al. (1963) provided an early analysis of hydromagnetic thermal convection. Wilkset al (1976) examined the buoyancy effects in hydromagnetic convection flows in strong magnetic fields. Soundalgekar and Takhar et al(1977) studied the MHD thermal convection flows pasta flat surface with applications in nuclear fusion blanket systems. Takhar and Soundalgekar(1980) investigated the influence of Eckert number on natural convection in magnetic fields. Revankar (1982) studied lateral mass flux effects in hydromagnetic convection. Takhar and Pop (1984) considered the influence of low thermal conductivity fluids on MHD thermoconvection flows past a two dimensional wedge geometry using shooting methods. Surma Devi et al (1986) modeled the hydromagnetic flow past a conical body using finite difference method. Thacker et al. (1988) considered the natural convection hydromagnetics in the vicinity of a disk configuration spinning in a vertical plane. Ram and Takhar (1993) analyzed the transient MHD free convection in a rotating fluid with Hall and ionslip current effects using a finite difference solver. Hossain et al. (1998) examined the effects of surface temperature oscillations on convection flows in transverse magnetic fields. Naroua et al. (2000) studied numerically the radiation effects on rotating transient MHD heat transfer. More recently Bég et al. (2001) studied the free convection hydromagnetics of a viscoelastic (second order) fluid in a non-Darcian porous medium. All these studies were concerned

\begin{tabular}{|c|c|}
\hline$\Phi \mathrm{p}$ & $\begin{array}{l}\text { dimensionless temperature of particle } \\
\text { phase }\end{array}$ \\
\hline$D a$ & Darcy number \\
\hline Fs & Forcheimmer number \\
\hline $\operatorname{Pr}$ & $\begin{array}{l}\text { Prandtl number } \\
\text { specific heat ratio }\end{array}$ \\
\hline$S k_{T}$ & inverse thermal Stokes number \\
\hline$S k_{m}$ & inverse hydrodynamic Stokes number \\
\hline$p_{L}$ & particle loading parameter \\
\hline B & dimensionless buoyancy parameter \\
\hline$H a$ & $\begin{array}{l}\text { Hartmann hydromagnetic number } \\
\text { viscosity ratio }\end{array}$ \\
\hline $\mathrm{Gr}$ & Grashof number \\
\hline$\Omega$ & $\begin{array}{l}\text { dimensionless particle-phase wall slip } \\
\text { parameter }\end{array}$ \\
\hline
\end{tabular}

with single phase hydrodynamic regimes. In many industrial and biophysical pheonomena multi-phase flows takes place. In such systems particles are transported in the main bulk of the fluid and often accompanied by simultaneous heat/mass transfer. These "dusty" flows have been reviewed by Marble (1970). Considerable interest has been shown in such flows in the engineering science community. Greenberg et al. (1973) provided an early study of vortex two phase flows. Peddieson (1975) investigated the gas particle fluid dynamics in aerodynamic flows past geometries in the presence of shock waves. Hwang and Chang (1988) simulated the twophase gas-particle flow in a solid rocket nozzle system using a computational method. Chamkha and Peddieson (1991) presented a fresh analysis of hydrodynamic boundary layers of particulate suspensions past flat surfaces. Leung et al. (1993) considered the interfacial two-phase flow hydrodynamics of bubbly liquids. Chang et al. (1996) implemented a state-of-the-art CFD algorithm to dilute gas-particle JPL nozzle flows. Satyamurthy et al. (1997) have studied two-phase hydrodynamics in liquid metal magnetohydrodynamic energy converters (LMMHD EC) of gravity type for electrical power generation. In these systems, vertical twophase flows consisting of steam and high density liquid metals like lead, lead alloys take place in the riser pipe. The design of optimum LMMHD $\mathrm{EC}$ and for scaling up of the system requires accurate modeling of the two-phase flow. The important parameter which governs the twophase flow was shown to be void fraction. Quantified void fraction and pressure profiles were compared with values based on two-phase bubble flow model and other well known empirical relations. It was noted that more robust mathematical models are needed for accurate prediction of void fraction for all ranges of liquid metal-nitrogen two-phase flows. Moukhalled et 
al. (2003) presented finite volume-based numerical solutions for incompressible/compressible multi-phase flow phenomena for subsonic, transonic, and supersonic regimes. Pressure was selected as the dependent variable in preference to density due to the significant changes in pressure at all sonic regimes as opposed to variations in density, which become very small at low Mach numbers. Several flow scenarios were considered including incompressible turbulent bubbly flow in a pipe, incompressible turbulent air-particle flow in a pipe, compressible dilute gas-solid flow over a flat plate, and compressible dusty flow in a converging diverging nozzle. Usha et al. (2005) studied computationally the particulate suspension flow through wavy-walled channels. A perturbation method was employed and the primitive variables were defined as series expansions with the wall amplitude as the perturbation parameter. The boundary conditions were applied at the mean surface of the channel and the first-order perturbation quantities numerically evaluated by solving the governing system of ordinary differential equations using a Runge-Kutta shooting quadrature. The wall shear stress and the flow separation/reattachment points were simulated and the influence of the volume fraction density of the particles was studied in detail. Variations of velocity and pressure of the particulate suspension flow with frequency of excitation were also considered. More recently Chamkha (1999) presented a continuum twophase fluid-particle model accounting for particle-phase stresses and a body force due to the presence of a magnetic field in a mathematical study of two-dimensional laminar hydromagnetic flow of a particulate suspension over a horizontal surface in the presence of a gravity field. Analytical solutions for the velocity distributions and the skin-friction coefficients of both phases were reported. The special cases of wall hydrodynamic (velocity) conditions corresponding to stationary and oscillatory velocity distributions were considered. Numerical evaluations of the analytical solutions were discussed and graphically presented to highlight special features of the solutions. The effects of the particle-phase stresses and the magnetic field were studied via representative results for the horizontal velocity profiles, fluid-phase displacement thickness, and the complete skinfriction coefficient for various combinations of the physical parameters. Magnetic field increased the fluid-phase skin-friction coefficient for various particulate volume fraction levels; conversely particle-phase viscous stresses were shown to depress fluid-phase skin-friction coefficient for various particle-to-fluid density ratios. Other applications of two-phase flows include steam turbines (1998), binary mixing processes (1998) and pulmonary hydrodynamics (2000). In the above studies the presence of porous media was not considered. Brenner (1993) has discussed the importance of such a medium in many chemical and energy systems applications. In cosmical fluid dynamics, the presence of debris and comet dust also exerts an important effect on transport processes in astro-plasmas. The present study therefore aims to develop a new mathematical model for two-phase hydromagnetic flow of a dusty particlesuspension system in a non-Darcian porous medium. The porous effects are simulated using a drag force model which has been applied successfully by Bég et al. (1996) to a plethora of engineering and geophysical flow problems. The bulk matrix resistance of the porous medium is modeled using the classical Darcian linear pressure drop formulation. The quadratic drag generated at higher Reynolds numbers is simulated using the Forcheimmer inertial model. The resulting drag force terms when combined constitute the Darcy-Forcheimmer resistance model. Bég et al. (2005) used this model recently in studying the rotating convection from a twodimensional plane to a thermally-stratified highporosity medium, the micropolar heat and mass transfer in a porous regime with Soret/Dufour diffusion effects was studied using the DarcyForcheimmer model by Bhargava et al. (2006) not only this the some is also done in channel using the non Newtonian fluid (2007).

In the recent past the authors focusing on the impact of viscous dissipation effects or thermal dissipation in Darcy-Forchheimer porous medium and Ghazian (2011) investigated analytically the impact of viscous dissipation in channel filled with porous medium he observed that the gap size between the porous region and the clear fluid directly affects the viscous dissipation due to change in the resistance of the fluid. A recent records reports the study of Mishra et al. (2012) on free convective fluctuating MHD flow past a vertical porous plate with variable temperature is cause of back flow and viscous dissipation accelerates the velocity.

Keeping in view the above literature our objective is to understand the impact different terminology for magneto-convection heat transfer of a two-phase, electrically-conducting, particlesuspension in a channel.

\section{Problem Formulation}

We consider the steady incompressible, magnetohydrodynamic fully-developed twophase flow and free convective heat transfer of a fluid-particle suspension in a two-dimensional parallel plate channel containing an isotropic, homogenous porous medium (as illustrated in Fig. 1). 


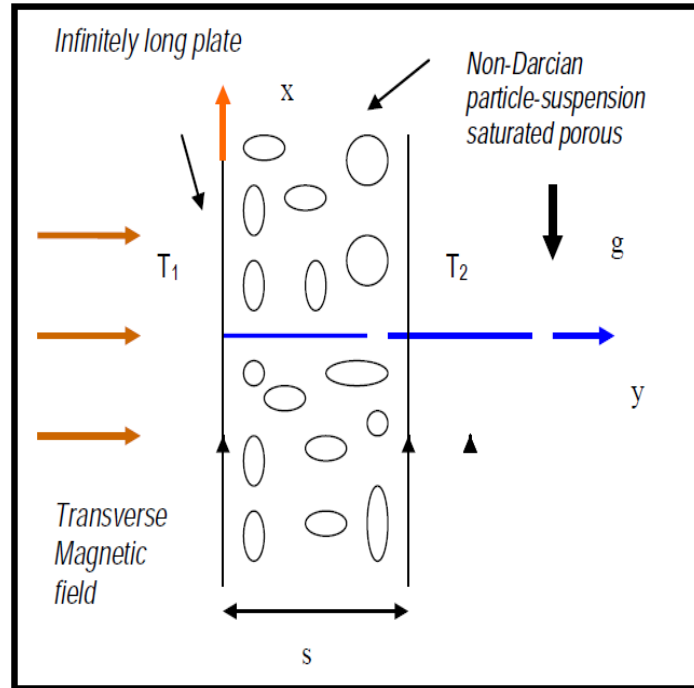

Fig. 1. Physical Model and Coordinate System

\section{Conservation of fluid momentum}

$$
\begin{gathered}
\mu \frac{d^{2} u}{d y^{2}}=\frac{d p}{d x}+\rho_{p} N\left(u-u_{p}\right)+\rho g+\frac{\mu}{K} u \\
+\frac{\rho b}{K} u^{2}+\frac{\sigma B^{2}}{\rho} u
\end{gathered}
$$

\section{Conservation of fluid energy}

$k \frac{d^{2} T}{d y^{2}}+\rho_{p} c_{p} N_{p}\left(T_{p}-T\right)=0$

\section{Conservation of particle momentum}

$\mu_{p} \frac{d^{2} u_{p}}{d y^{2}}+\rho_{p} N\left(u-u_{p}\right)+\rho_{p} g$

\section{Conservation of particle energy}

$$
\rho_{p} c_{p} N_{p}\left(T_{p}-T\right)=0
$$

All parameters relating to Eqs. (1) to (4) are defined in the nomenclature. The conservation of mass in both phases is also identically satisfied. Pressure gradient appears only in the fluid phase momentum equation. Following Al-Subaie and Chamkhawe eliminate this term from the momentum equation, by re-defining the transport equations at a reference point within the channel. Denoting:

$\mathrm{u}=0, \quad \mathrm{~T}=\mathrm{T}_{\mathrm{o}}, \quad \rho=\rho_{0}, \quad \mu=\mu_{0}$,

$\sigma=\sigma_{0}, \quad u_{p}=\sigma_{p 0}, \quad T_{p}=T_{p 0}$,

$\rho_{\mathrm{p}}=\rho_{\mathrm{p} 0}, \mu_{\mathrm{p}}=\mu_{\mathrm{p} 0}$

and implementing the Boussinesq approximation , Eq. (1) transforms to:

$$
\begin{aligned}
& \frac{\mu_{0}}{\rho_{0}} \frac{d^{2} u}{d y^{2}}+\beta^{*} g\left(T-T_{0}\right)-\frac{\rho_{p_{0}}}{\rho_{0}} N\left(u-u_{p}\right)+ \\
& \frac{\rho_{p_{0}}}{\rho_{0}} g=\frac{\mu_{0}}{K} u+\frac{\rho_{0} b}{K} u^{2}+\frac{\sigma_{0} B_{0}^{2}}{\rho} u
\end{aligned}
$$

The particles are assumed to be homogenous (i.e. number density of the particles is constant throughout the motion), discrete and nonconducting. Interaction between the particles is also ignored. The $\mathrm{x}$-direction is directed along the longitudinal axis of the plates and the y direction is normal to this. For infinitely long channel walls the problem reduces to a one-dimensional flow since dependence on the $\mathrm{y}$ - direction swamps dependence of variables on the $\mathrm{x}$-direction. Zero electrical field is assumed throughout the flow regime and the effects of chemical reaction, mass transfer and radiation between the particles and fluid is neglected. The porous medium is assumed to be in local thermal equilibrium and dispersion and stratification effects are ignored. The appropriate transport equations for the flow can be shown to take the form as the following ordinary differential equations, where $\mathrm{y}$ is the only independent variable:

The governing equations are now (5), (2), (3) and (4) and the appropriate boundary conditions at either channel wall are defined by:

\section{For the fluid phase:}

$u(0)=u(s)=0 ; T(0)=T_{1} ; T(s)=T_{2}$

\section{For the particle phase:}

$u_{p}(0)=\omega \frac{d u_{p}(0)}{d y}-\frac{g}{N} ; u_{p}(s)=-\omega \frac{d u_{p}(s)}{d y}-\frac{g}{N}$

where all terms are again defined in the nomenclature at the end of the paper. The current problem is therefore a sixth order system of ordinary differential equations with six robust boundary conditions. The velocity boundary conditions (5a) correspond to no-slip conditions for the fluid phase at the channel walls. Conditions (5b) are infact deduced from an analogy to rarified gas dynamics Hayes et.al (1970) and constitute generalized wall boundary conditions. This approach has been adopted by many researchers and is valid both numerically and experimentally turbines Marble (1970), Alsubaie (2003)

\section{Transformation of Model}

To facilitate a numerical solution to the system (5), (2), (3), (4) and (5a,b), we now introduce a set of non-dimensional transformations and thermofluid numbers, viz:

$$
\begin{gathered}
y=s \eta, u=\frac{\mu}{\rho s} U, u_{p}=\frac{\mu}{\rho s} U_{p}, \\
T=\left[T_{2}-T_{0}\right] \Phi+T_{0}
\end{gathered}
$$


$T_{0}=\frac{\left[T_{1}+T_{2}\right]}{2}, \Lambda=\frac{\mu_{p}}{k \mu}, T_{p}=\left[T_{2}-T_{0}\right] \Phi+$

$T_{0}, D a=\frac{K}{S^{2}}, F_{S}=\frac{b}{s}, \operatorname{Pr}=\frac{\mu c}{k}, \gamma=\frac{c_{p}}{c}, S k_{T}=$

$\frac{\rho N_{T} S^{2}}{\mu}, S k_{m}=\frac{\rho N s^{2}}{\mu}, p_{L}=\frac{\rho_{p}}{\rho}$

$H a=\sqrt{\frac{\sigma B^{2} s^{2}}{\mu}}, B=\frac{g \rho^{2} s^{3}}{\mu^{2}}, G r=\frac{g \beta^{*} \rho^{2} s^{3}\left[T_{2}-T_{0}\right]}{\mu^{2}}$

where all non-dimensional quantities are defined in the nomenclature. The transport equations now become:

$\frac{d^{2} U}{d \eta^{2}}+G r \Phi-S k_{m} p_{L}(U-U)+p_{L} B-\frac{1}{D a} U-$

$\frac{F S}{D a} U^{2}-H a^{2} U=0$

$\frac{1}{P r} \frac{d^{2} \Phi}{d \eta^{2}}+S k_{T} p_{L} \gamma\left(\Phi_{\mathrm{p}}-\Phi\right)=0$

$\Lambda \frac{d^{2} U_{p}}{d \eta^{2}}+S k_{m}\left(U-U_{p}\right)-B=0$

$S k_{T} \gamma\left(\Phi_{\mathrm{p}}-\Phi\right)=0$

with corresponding boundary conditions:

For the fluid phase:

$U(0)=U(1)=0 ; \Phi(0)=-1 ; \Phi(1)=1$

For the particle phase:

$U(0)=\Omega \frac{d U_{p}(0)}{d \eta}-\frac{B}{S k_{m}}$

$U(1)=-\Omega \frac{d U_{p}(1)}{d \eta}-\frac{B}{S k_{m}}$

and the new parameter, $\Omega$ i.e. dimensionless particle-phase wall slip parameter, is defined in the nomenclature. The system (6) to (9) with conditions $(10 \mathrm{a}, \mathrm{b}, \mathrm{c})$ defines a well-posed twopoint boundary value problem which yields numerical solutions for a wide range of all the thermophysical parameters. Prior to embarking on a numerical solution we shall first describe a number of important special cases relating to the general transformed, dimensionless model.

\section{Special Cases for the Flow Model}

Case I: Hydromagnetic Inviscid ParticleSuspension Free Convection Flow in a Darcy-Forcheimmer Porous Channel

When the viscosity ratio vanishes i.e. $\Lambda \rightarrow 0$, the fluid suspension loses viscosity i.e. becomes inviscid, a regime usually encountered in aerodynamic flows. This has a major influence on only the particle-phase momentum Eq. (8) which now reduces to an algebraic equation:

$S k_{m}\left(U-U_{p}\right)-B=0$
The other conservation equations are not affected.

Case II: Non-Conducting ParticleSuspension Free Convection Flow in a Darcy-Forcheimmer Porous Channel

When the Hartmann number is set to zero i.e. Ha $\rightarrow 0$, hydromagnetic drag is negated and the flow regime becomes non-conducting. In this case only the fluid phase momentum Eq (6) is affected, contracting to :

$$
\begin{aligned}
& \frac{d^{2} U}{d \eta^{2}}+G r \Phi-S k_{m} p_{L}\left(U-U_{p}\right)+p_{L} B-\frac{1}{D a} U- \\
& \frac{F S}{D a} U^{2}=0
\end{aligned}
$$

Case III: Non-Conducting ParticleSuspension Free Convection Flow in a Darcian Porous Channel

Case II may be further modified for the scenario where the Forcheimmer number is set to zero so that the quadratic drag force term vanishes in Eq (12). The momentum equation thereby reduces to:

$$
\begin{aligned}
& \frac{d^{2} U}{d \eta^{2}}+G r \Phi-S k_{m} p_{L}\left(U-U_{p}\right)+p_{L} B-\frac{1}{D a} U= \\
& 0
\end{aligned}
$$

which physically corresponds to the Darcian convection regime. Once again the other transport equations are unaffected.

\section{Case IV: Non-conducting Particle- Suspension Free Convection Flow in a Non-Porous Channel}

For the case of infinite permeability, $\mathrm{Da} \rightarrow \infty$, and in Eq (13) the Darcian bulk impedance term vanishes, so that the flow domain is now one of natural convection of a particle-fluid suspension in a non-porous channel. Equation (13) shrinks now to:

$\frac{d^{2} U}{d \eta^{2}}+G r \Phi-S k_{m} p_{L}\left(U-U_{p}\right)+p_{L} B=0$.

Case V: Non-conducting ParticleSuspension Forced Convection Flow in a Non- Porous Channel

For the above four special cases, the fluid velocity field is coupled to the temperature field via the buoyancy term in (6). In addition there is a buoyancy term due to particle-fluid effects in (6). For forced convection, Gr $\rightarrow 0$, and this decouples the fluid and temperature variables in (6) and (7). We can therefore modify (14) to reduce the fluid phase momentum equation yet further, to give:

$\frac{d^{2} U}{d \eta^{2}}-S k_{m} p_{L}\left(U-U_{p}\right)+p_{L} B=0$ 
We note buoyancy is still present in the last term in Eq. (15) which is clearly independent of the temperature field.

Several of these special cases are considered in the numerical computations to be provided next

\section{Numerical Solutions by the Finite Element Method}

The governing equations amount to a set of nonlinear, coupled ordinary differential equations with corresponding boundary conditions. The finite element method $\operatorname{Rao}(1982)$ is a versatile numerical solver which has been applied in many areas of fluid dynamics including combustion, reactive aerodynamics, solar engineering systems, rheology, ocean wave dynamics, river hydraulics and flame acoustics etc. A finite element solution of the transformed equations (6) to (9) with $(10 \mathrm{a}, \mathrm{b}, \mathrm{c})$, encompasses initially the derivation of a variational formulation. Further details are available in the monograph by Bathe Eq (6) Bathe (1996). The basic stages of this technique are as follows:

\section{Discretization of the domain into elements:}

(i) The whole domain is divided into a finite number of sub domains, which is called the discretization of the domain. Each sub domain is called an element. The collection of elements is then denoted the finite-element mesh. The intersection of any two elements is termed an inter-element boundary. The intersection points are called the global nodes.

(ii) The nodes and elements are numbered.

(iii) The geometric properties needed for the problem are generated, which indicates the position of the elements.

\section{Derivation of element equations:}

(i) A typical element is isolated from the mesh and the variational formulation of the given problem over the typical element is constructed.

(ii) An approximate solution of the variational problem is assumed. Substituting it into 2.(i), the element equations are made.

(iii) The element matrix or stiffness matrix is constructed by using the element interpolation functions. The interpolation functions depend on the type of element (geometry, number of nodes and number of primary unknowns per node). These functions have to be derived or selected and are not already available in the literature.

\section{Assembly of Element Equations:}

The algebraic equations so obtained are assembled by imposing the interelement continuity conditions (i.e. the value of the nodal variables at the nodes are identical for two or more elements). This yields a large number of algebraic equations known as the global finite element model, which governs the whole domain.

\section{4- Imposition of boundary Conditions:}

The initial and final boundary conditions are imposed on the assembled equations.

\section{Solution of assembled equations:}

The assembled equations are solved by any of the numerical technique viz. Gaussian Elimination, Gauss-Seidel, LU decomposition etc.

For computational purposes and without loss of generality, $\infty$ has been fixed as 1 . The whole domain is divided into a set of 81 line elements of equal width, each element being two-noded.

\section{Variational formulation:}

The variational form associated with differential equations (6)-(8), Eq (9) is a linear expression and not considered in this stage of the solution] over a typical two noded linear element

$$
\begin{aligned}
& \left(\eta_{e}, \eta_{e+1}\right) \text { is given by: } \\
& \int_{\eta_{e}}^{\eta_{e+1}} w_{1}\left\{\frac{d^{2} U}{d \eta^{2}}+\operatorname{Gr} \Phi-S k_{m} p_{L}\left(U-U_{p}\right)+\right. \\
& p L B-1 D a U-F S D a U 2-H a 2 U d \eta=0
\end{aligned}
$$

Where, $w_{1}, w_{2}$ and $w_{3}$, are arbitrary test functions and may be viewed as the variation in $U, \Phi$ and $U_{p}$, respectively.

\section{Finite element formulation:}

The finite element model may be obtained from Eqs. (6)-(8) by substituting finite element approximations of the form:

$U=\sum_{j=1}^{2} U_{j} \psi_{j}, \Phi=\sum_{j=1}^{2} \Phi_{j} \psi_{j}, U_{p}=$ $\sum_{j=1}^{2} U_{p j} \psi_{j}$

With, $w_{1}=w_{2}=w_{3}=U_{p j} \psi_{i} \quad, \quad(\mathrm{i}=1,2)$, The parameters, $\psi_{i}$, are the shape functions for a typical element $\left(\eta_{e}, \eta_{e+1}\right)$, and are taken as:

$\psi_{1}^{(e)}=\frac{\eta_{e+1}-\eta}{\eta_{e+1}-\eta_{e}}, \psi_{2}^{(e)}=\frac{\eta-\eta_{e}}{\eta_{e+1}-\eta_{e}} \quad \eta_{e} \leq \eta \leq \eta_{e+1}$

The finite element model of the equations thus formed is given by:

$$
\left[\begin{array}{lll}
{\left[K^{11}\right]} & {\left[K^{12}\right]} & {\left[K^{13}\right]} \\
{\left[K^{21}\right]} & {\left[K^{22}\right]} & {\left[K^{23}\right]} \\
{\left[K^{31}\right]} & {\left[K^{32}\right]} & {\left[K^{33}\right]}
\end{array}\right]\left[\begin{array}{l}
\{U\} \\
\{\Phi\} \\
\left\{U_{p}\right\}
\end{array}\right]=\left[\begin{array}{l}
\left\{b^{1}\right\} \\
\left\{b^{2}\right\} \\
\left\{b^{3}\right\}
\end{array}\right]
$$

Where, $\left[K^{m n}\right]$ and $\left[b^{m}\right](\mathrm{m} . \mathrm{n}=1,2,3,4,5)$, are the matrices of order $2 \times 2$ and $2 \times 1$ respectively. All these matrices may be defined as follows: 


$$
\begin{aligned}
& K_{i j}^{11}=-\int_{\eta_{e}}^{\eta_{e+1}} \frac{d \psi_{i}}{d \eta} \frac{d \psi_{j}}{d \eta} d \eta-S k_{m} p_{L} \int_{\eta_{e}}^{\eta_{e+1}} \psi_{i} \psi_{j} d \eta \\
& -\frac{1}{D a} \int_{\eta_{\mathrm{e}}}^{\eta_{\mathrm{e}}+1} \psi_{\eta_{\mathrm{i}}} \psi_{\mathrm{j}} \mathrm{d} \eta \\
& -\frac{F s}{D a} \overline{U_{1}} \int_{\substack{\eta_{e} \\
\eta_{e+1}}}^{\eta_{e+1}} \psi_{i} \psi_{1} \psi_{j} d \eta \\
& -\frac{F s}{D a} \overline{U_{2}} \int_{\eta_{e}}^{\eta_{e+1}} \psi_{i} \psi_{2} \psi_{j} d \eta \\
& -H a^{2} \int_{\eta_{e}}^{\eta_{e+1}} \psi_{i} \psi_{j} d \eta \\
& K_{i j}^{12}=G r \int_{\eta_{e}}^{\eta_{e+1}} \psi_{i} \psi_{j} d \eta, \\
& K_{i j}^{13}=S k_{m} p_{L} \int_{\eta_{e}}^{\eta_{e+1}} \psi_{i} \psi_{j} d \eta \\
& K_{i j}^{21}=0 \text {, } \\
& K_{i j}^{22}=-\int_{\eta_{e}}^{\eta_{e+1}} \frac{d \psi_{i}}{d \eta} \frac{d \psi_{j}}{d \eta} d \eta \\
& K_{i j}^{23}=0 \\
& K_{i j}^{31}=S k_{m} \int_{\eta_{e}}^{\eta_{e+1}} \psi_{i} \psi_{j} d \eta \\
& K_{i j}^{32}=0 \\
& K_{i j}^{33}=-\Lambda \int_{\eta_{e}}^{\eta_{e+1}} \frac{d \psi_{i}}{d \eta} \frac{d \psi_{j}}{d \eta} d \eta-S k_{m} \int_{\eta_{e}}^{\eta_{e+1}} \psi_{i} \psi_{j} d \eta \\
& b_{i}^{1}=-p_{L} B-\left(\psi_{i} \frac{d U}{d \eta}\right)_{\eta_{e}}^{\eta_{e+1}}, b_{i}^{2}=-\left(\psi_{i} \frac{d \Phi}{d \eta}\right)_{\eta_{e}}^{\eta_{e+1}}, \\
& b_{i}^{3}=B-\Lambda\left(\psi_{i} \frac{d U_{p}}{d \eta}\right)_{\eta_{e}}^{\eta_{e+1}}
\end{aligned}
$$

Where, $\bar{U}=\sum_{i=1}^{2} \bar{U}_{l} \psi_{i}$, Each element matrix is of the order $6 \times 6$, Since the whole domain is divided into a set of 81 line elements, following assembly of all the elements equations we obtain a matrix of order $246 \times 246$, This system of equations as obtained after assembly of the elements equations is non-linear therefore an iterative scheme has been used to solve it. The system is linearized by incorporating the function $\bar{U}$, which is assumed to be known. After applying the given boundary conditions only a system of equations remains for the solution which has been solved using Gauss elimination method by maintaining an accuracy of 0.0005 . We note that the present model is restricted to steady flow. For the transient case, relaxation time and Strouhal number effects have to be considered, and such a study is currently under development by Bég et al. (2006). The finite element technique overcomes the shortcoming of the traditional variational method and is highly efficient computationally. This method has been applied by the authors in many nonlinear flow problems including biomagnetic micropolar porous flow (2006), micropolar stagnation point convection flow (1998), the micropolar flow between rotating disks (2001).

\section{Discussion of Results}

The 11 parameters governing the flow and heat transfer in the porous channel regime are: Prandtl number (Pr), Grashof free convection number (Gr), Hartmann hydromagnetic number (Ha), inverse Stokes number $\left(\mathrm{Sk}_{\mathrm{m}}\right)$, Darcy number (Da), Forcheimmer number (Fs), particle loading parameter $\left(\mathrm{P}_{\mathrm{L}}\right)$, buoyancy parameter (B), temperature inverse Stokes number $\left(\mathrm{Sk}_{\mathrm{T}}\right)$, Viscosity ratio $(\Lambda)$, specific heat ratio $(\gamma$

).Computations have been performed for selected thermophysical parameters, for conservation of space, for example Grashof free convection number $(\mathrm{Gr})$, Hartmann hydromagnetic number $(\mathrm{Ha})$, inverse Stokes number $\left(\mathrm{Sk}_{\mathrm{m}}\right)$, Darcy number (Da), Forcheimmer number (Fs), particle loading parameter $\left(\mathrm{p}_{\mathrm{L}}\right)$, buoyancy parameter $(\mathrm{B})$ on the fluid-phase velocity and particle-phase velocity. Here we dwell therefore on the response of the velocities to variations in the control parameters. In a subsequent paper Rawat (2010),, we shall consider in detail the effects of such parameters on the temperature profiles also. From equation Rawat (2010), the $\Phi$ value (dimensionless temperature of fluid phase) and the $\Phi_{\mathrm{p}}$ (dimensionless temperature of particle phase) will be equivalent, therefore only a numerical solution for $\Phi$ is needed, as discussed above in the methodology.

We have prescribed the following default values for the thermophysical parameters:

$\operatorname{Pr}=1, \mathrm{Gr}=1, \mathrm{Ha}=1, \mathrm{Sk}_{\mathrm{m}}=1, \mathrm{Da}=1, \mathrm{Fs}=1$, $\mathrm{P}_{\mathrm{L}}=1, \mathrm{~B}=1, \mathrm{Sk}_{\mathrm{T}}=1, \Lambda=1, \gamma=1$.

In Figure 2, we have plotted the distribution of $U$ i.e. dimensionless $\eta$-direction fluid phase velocity versus $\eta$ for various Grashof numbers i.e. free convection parameter. As $\mathrm{Gr}$ increases from 0 through 100, 200, 300 and 500, $\mathrm{U}$ values are

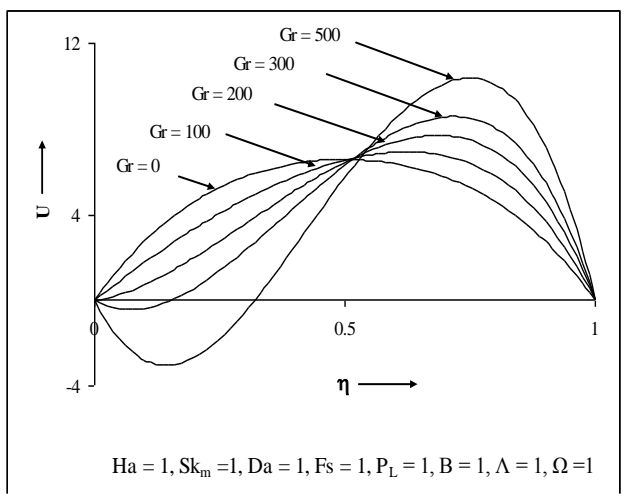

Fig. 2. $U$ versus $\eta$ for various $G r$ values

initially decreased in magnitude for the regime 0 $<\eta<0.5$. For higher Gr values i.e. 300 and 500 
which correspond to strong buoyancy the $\mathrm{U}$ values in fact become negative indicating low reversal over the range $0<\eta<0.125$ and $0<\eta<$ 0.3 respectively; However we observe that in the second half of the channel, $1.0<\eta<0.5$, the $U$ values remain positive and in fact increase with buoyancy i.e. the maximum velocity in the channel corresponds to $\mathrm{Gr}=500$ at $\eta \sim 0.7$. It is interesting to note also that in the forced convection case, $\mathrm{Gr}=0$, the velocity profile is parabolic and perfectly symmetrical about the channel centre line at $\eta \sim 0.5$.Increasing buoyancy effects push profiles downwards over the first half of the channel and upwards over the second half of the channel. All profiles descend to zero at both walls of the channel in accordance with the zero velocity boundary conditions there. Figure 3 illustrates the distribution of dimensionless $\eta$-direction fluid phase velocity, $U$, with $\eta$ for various Hartmann (hydromagnetic) numbers, Ha. The profiles in this plot are all symmetrical parabolas about the channel centre line, and correspond to weak buoyancy effects $(\mathrm{Gr}=1)$. Ha defines the relative effects of the magnetic retarding force to the viscous force. A rise in Ha clearly decreases $U$ values from a peak value of 6.8 for $\mathrm{Ha}=0$ (at the channel centre-line, $\eta=0.5$ ) to approximately 5 for $\mathrm{Ha}=3$, to 1.4 for $\mathrm{Ha}=5$ and to 0.2 for $\mathrm{Ha}=20$. The case of $\mathrm{Ha}=$ 0 corresponds to non-magnetic i.e. hydrodynamic flow and heat transfer, where the fluid is not electrically-conducting. A magnetic field applied in the $\eta$-direction, therefore generates a resistive force perpendicular to this direction i.e. in the direction of flow (longitudinal) which decelerates the movement of the fluid in the channel. As a result the minimum velocities correspond to the maximum Ha values and vice versa. Magnetic fields therefore can be imposed normal to a channel flow to control flow velocities in energy generation devices etc. The graph again corresponds to non-Darcian channel flow with

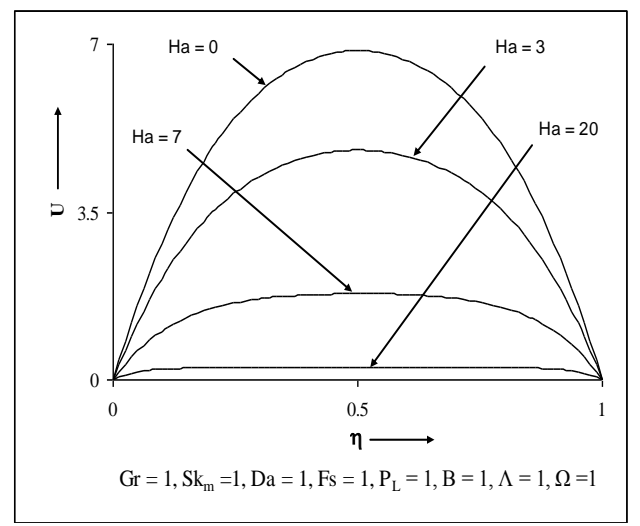

Fig. 3. $U$ versus $\eta$ for various $H a$ values

weak buoyancy. We also note that in all the computations reported here the viscosity ratio, $\Lambda$, has been fixed at unity implying that the both fluid and particle phases possess the same viscosity. Computations have been performed (not reported here for brevity) for the effects of $\Lambda$ which when increasing implies that particle phase viscosity coefficient rises and the suspension becomes more viscous causing a reduction in velocities and also skin friction values.

Figure 4 shows the variation of the particle-phase velocity $U_{p}$ with Hartmann number $(\mathrm{Ha})$. An increase in Ha causes an decrease in $U_{p}$ values (which are again symmetric about the centre line of the channel). The dimensionless particle-phase wall parameter, $\Omega$, is set to unity in both boundary conditions $(44 \mathrm{~b}, \mathrm{c})$ and with buoyancy parameter (B) and momentum inverse Stokes number $\left(\mathrm{Sk}_{\mathrm{m}}\right)$ also equal to unity, which therefore reduce to : $U_{p}(0)=\frac{d U_{p}(0)}{d \eta}-1 \lim _{\delta x \rightarrow 0}$ and $U_{p}(1)=-\frac{d U_{p}(1)}{d \eta}-1$ at the left wall $(\eta=0)$ and the right wall of the channel $(\eta=1)$ respectively. The least value of $U_{p}$ is observed for $\mathrm{Ha}=20$ (maximum magnetic field strength case) and occurs at $\eta=0.5$ and is equal to -63 approximately. For $\mathrm{Ha}=0$ (non-conducting case) the least value is about -58 . In all cases the particle phase velocities are in fact negative. The maximum values for all values of Ha correspond to the wall where due to symmetry they are identical for each case of Ha. Clearly therefore magnetic field has a much more pronounced effect on the fluid phase velocities (Fig. 3) than on the particle-phase velocities. This is logical since the U-conservation equation is directly affected by $\mathrm{Ha}$ via the Lorentz body force term in (40), $H a^{2} U$, and this via presence of the $U$ term in the $U_{p}$ momentum equation (42), $S k_{m}\left(U-U_{p}\right)$, causes an indirect influence on the particle-phase velocity field.

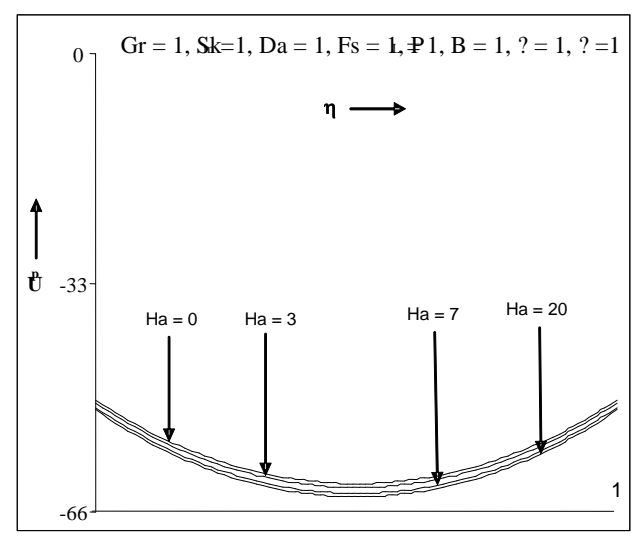

Fig. 4. $U_{p}$ versus $\eta$ for various $H a$ values

The influence of momentum (hydrodynamic) inverse Stokes number, $\mathrm{Sk}_{\mathrm{m}}$, on $\mathrm{U}$ and $\mathrm{U}_{\mathrm{p}}$ profiles versus $\eta$ are depicted in Fig. 5 and $6 \mathrm{Sk}_{\mathrm{m}}$ therefore signifies the hydrodynamic coupling between the fluid and the particle phases. As $\mathrm{Sk}_{\mathrm{m}}$ increases. therefore there is a 


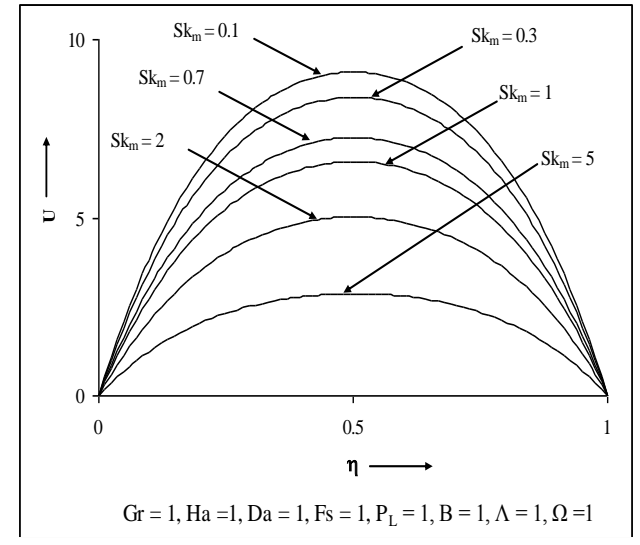

Fig. 5. $U$ versus $\eta$ for various $S k_{m}$ values

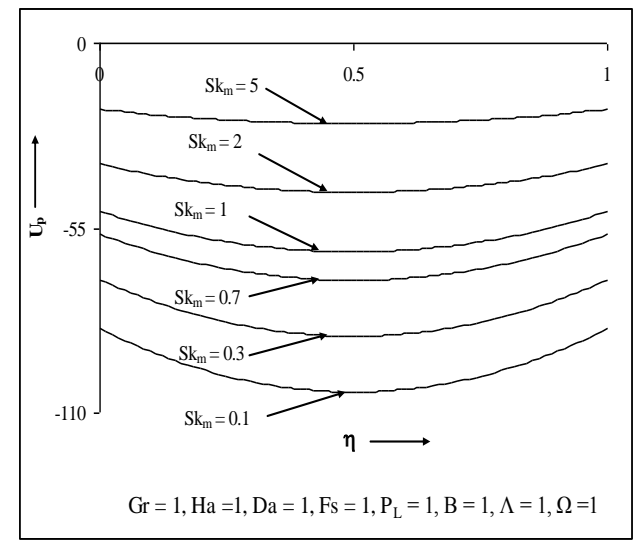

Fig. 6. $U_{p}$ versus $\eta$ for various $S k_{m}$ values

correspondingly greater transfer of momentum from the fluid phase to the particle phases, which causes a decreases in $U$ (fluid phase) velocity throughout the channel, as shown in figure 5. Peak values of $U$ therefore occur for the least value of $\mathrm{Sk}_{\mathrm{m}}$ i.e. 0.3 (more momentum is transferred to the fluid than the particle phase) and the lowest $U$ values arise when $\mathrm{Sk}_{\mathrm{m}}$ reaches a maximum value of 5 (more momentum transferred to the particle phase). For the profile $\mathrm{Sk}_{\mathrm{m}}=1$ an equal momentum allocation is achieved between both phases. The maximum $\mathrm{U}$ velocities always occur at the centre-line of the channel (values are zero at both channel walls), and are 9 and 2 approximately for $\mathrm{Sk}_{\mathrm{m}}=0.3$ and 5 respectively. In consistency with the $\mathrm{U}$ profiles, the Up profiles are seen in figure 6 to increase considerably i.e. become progressively less negative as $\mathrm{Sk}_{\mathrm{m}}$ increases from 0.1 through $0.3,0.7,1,2$ to 5 . Profiles also become increasingly flatter as $\mathrm{Sk}_{\mathrm{m}}$ increases. The minimum value computed for $U_{p}$ is approximately -105 at $\eta=0.5$ (for $\mathrm{Skm}=0.1$ for which $90 \%$ of the momentum is transferred to the fluid) and the maximum value of -15 for $\mathrm{Sk}_{\mathrm{m}}=5$ (five times as much momentum transferred to particle phase as to the fluid phase).

The influence of the Darcy number, Da, on $\mathrm{U}$ and $\mathrm{U}_{\mathrm{p}}$ distributions are illustrated in Fig. 7 and 8 This parameter is directly proportional to the permeability of the porous regime i.e. $\mathrm{K}$ and

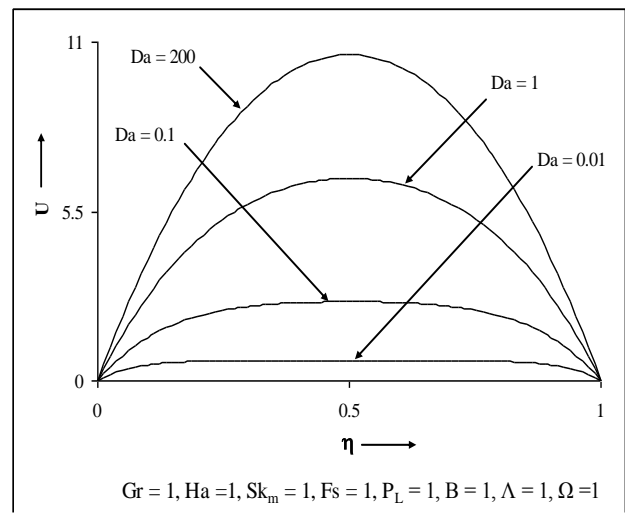

Fig. 7. $U$ versus $\eta$ for various $D a$ values

occurs as a denominator in both the Darcian drag and Forchheimer drag forces in the fluid phase momentum conservation Eq. (6) i.e. $\frac{1}{D a} U$. As $\mathrm{Da}$ increases, the bulk matrix (Darcian) drag decreases and therefore an increase in velocity, $\mathrm{U}$ accompanies a rise in Da from 0.001 through 0.1, 1 to 200 (highly permeable). $D a=0.01$ physically relates therefore in figure 7 to the least permeable scenario corresponding to the maximum matrix resistance of the solid particles in the flow regime. In a geophysical context these would be generated by for example the geomaterial structure through which the fluidparticle suspension is flowing. All profiles for $\mathrm{Da}$ $=0.1,1$ and 200 are symmetric parabolas about the centre line of the channel and velocities at either wall are zero (no-slip condition).

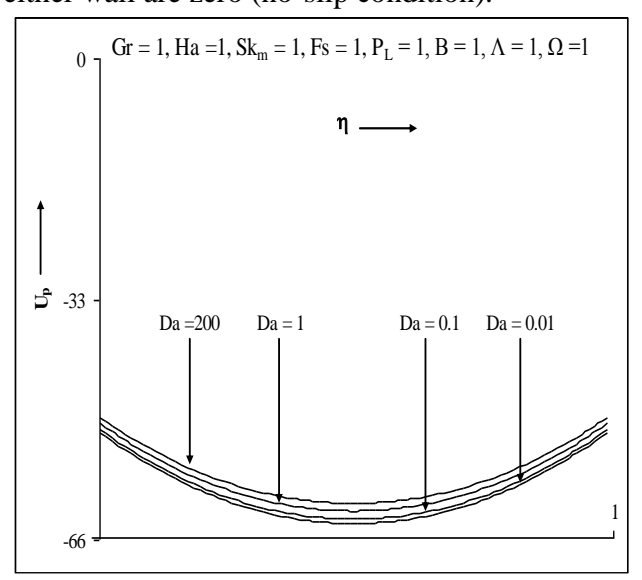

Fig. 8. $U_{p}$ versus $\eta$ for various $D a$ values

For $\mathrm{Da}=0.01$ the profile is almost horizontal and peak velocity plummets to 0.4 at $\eta=0.5$. In figure 8 we observe that the particle phase velocity is also increased by a rise in Darcy number from 0.01 , through 0.1 and 1 to 200 i.e. $\mathrm{U}_{\mathrm{p}}$ values become less negative. However the increase is much less marked which is due to the indirect effect that the Darcy number incurs on the particle phase momentum field. Da does not occur in this momentum equation, and only in 
the fluid momentum conservation equation. The coupling of the $U$ and $U_{p}$ fields indirectly allows the Darcy number to effect the particle phase velocity, $\mathrm{U}_{\mathrm{p}}$, explaining the much less pronounced effects. As with all other computations a Prandtl number of unity has been implemented.

In Figs. 9 and 10 we have plotted the variation of $\mathrm{U}$ and $\mathrm{U}_{\mathrm{p}}$ with the quadratic porous parameter i.e. Forchheimer number, Fs. For the case of Fs $=0$ the regime is Darcian. As Fs is increased to 1, 5 and then 50 (strong inertial drag), the velocity $\mathrm{U}$ is dramatically decreased as witnessed in figure 9 .

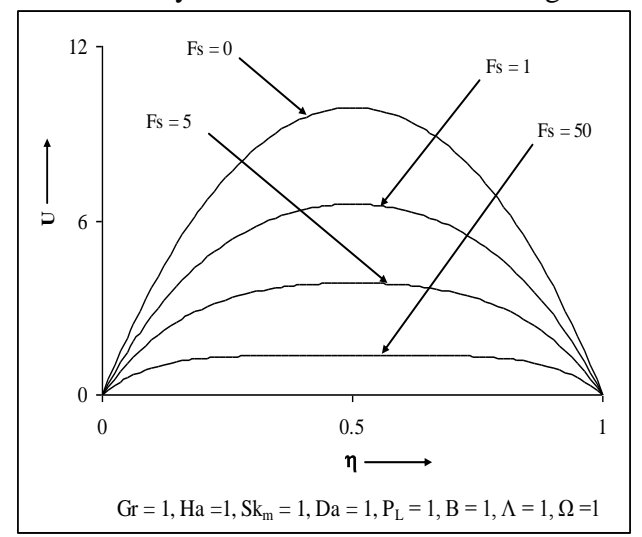

Fig. 9. $U$ versus $\eta$ for various $F s$ values

With $\mathrm{Da}=1$ in the computations a rise in Fs from 1 to 50 implies a fifty fold increase in quadratic impedance in the term, $\frac{F s}{D a} U^{2}$. The profiles for Fs $=0,1$, and 5 are symmetric parabolas, however as with the case of magnetic field (figure 3 ), for high values of Fs the profile becomes greatly flattened. Velocity gradients therefore are minimized at the centerline of the channel for Fs $=50$ as are velocities. A maximum $U$ value of approximately 10 is observed at the centerline of the channel for $\mathrm{Fs}=0$; the minimum value is roughly 1 for $F s=50$.

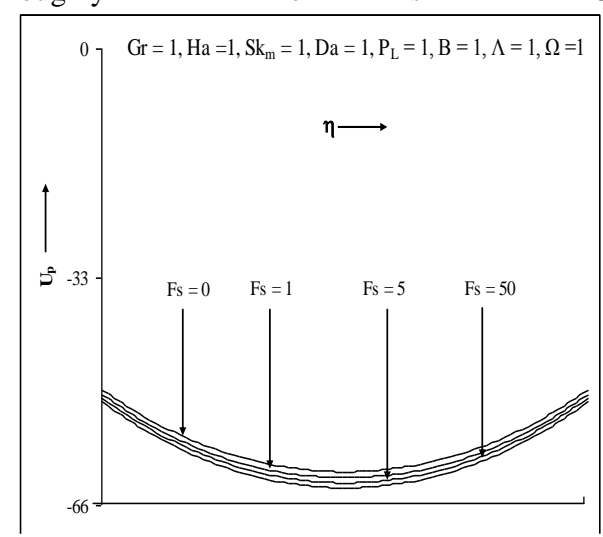

Fig. 10. $U_{p}$ versus $\eta$ for various $F s$ values

As expected a rise in Fs decreases the values of particle phase velocity i.e. $U_{p}$ i.e. values become more negative, as seen in figure 10. As before with the Da and Ha effects, the influence of the same range of increase of Fs on the $U_{p}$ values is much less distinct than for the $U$ values. As with the other body force effects i.e. magnetic field and Darcian bulk matrix drag, the influence of Forchheimer inertial drag is only experienced indirectly by the particle phase momentum field via coupling to the fluid phase momentum field through the term, $S k_{m}\left(U-U_{p}\right)$ in equation (8).

It is important to note that primarily deceleration in the channel is experienced by the fluid phases rather than the particle phases due to inertial drag effects, which may be of significance in filtration applications in chemical engineering.

The effects of the particle loading parameter, $\mathrm{P}_{\mathrm{L}}$, on the fluid phase and particle phase velocities is shown in Fig. 11 and 12. $p_{L}=\frac{\rho_{p}}{\rho}$ which represents the ratio of the densities of the particle and fluid phases. An increase in $\mathrm{P}_{\mathrm{L}}$ clearly boosts the fluid phase velocity, $\mathrm{U}$, which rises from approximately 1 for $\mathrm{P}_{\mathrm{L}}=0.1$ to 13 for $\mathrm{P}_{\mathrm{L}}=3$. Profiles are generally smoothly parabolic and symmetric about $\eta \sim 0.5$, with the exception of $\mathrm{P}_{\mathrm{L}}$ $=0.2$ for which the density of the fluid is much greater than the particle phase. In figure 12 particle-phase velocity, Up is seen to increase marginally with a rise in $\mathrm{P}_{\mathrm{L}}$ from 0.1 through 0.5 , 1 , 2 and

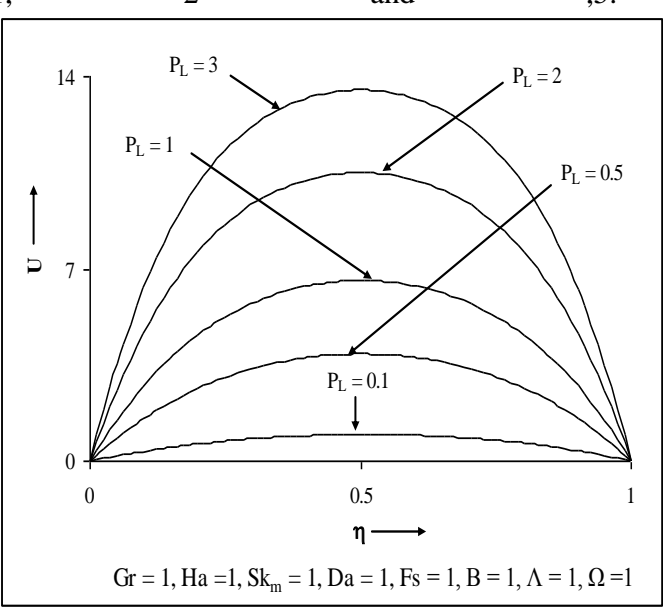

Fig. 11. $U$ versus $\eta$ for various $P_{L}$ values

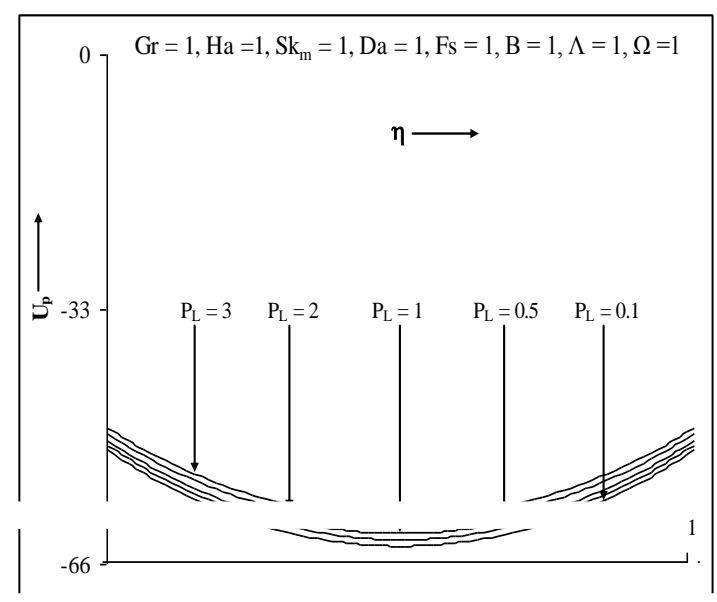

Fig. 12. $U_{p}$ versus $\eta$ for various $P_{L}$ values 
Hence particle phase velocities become less negative as $\mathrm{P}_{\mathrm{L}}$ increases.

Finally in Fig. 13 and 14 we have provided the distributions of $U$ and $U_{p}$ for various gravitational buoyancy parameter, $B=\frac{\rho^{2} g s^{3}}{\mu^{2}}$. A substantial

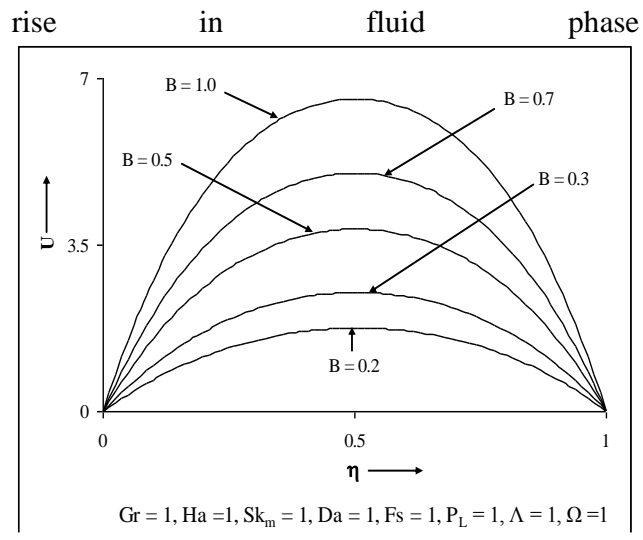

Fig. 13. $U$ versus $\eta$ for various $B$ values

Velocity, U, occurs as B increases from 0.2 through $0.3,0.5,0.7$ and to 1.0. Buoyancy therefore substantially boosts the fluid momentum and accelerates the flow. Peak U velocity occurs for $\mathrm{B}=0.7$ and the minimum peak velocity (in both cases a the centre of the channel) for $\mathrm{B}=0.2$ (weak buoyancy). All profiles are symmetric parabolas and steepest at the channel walls.

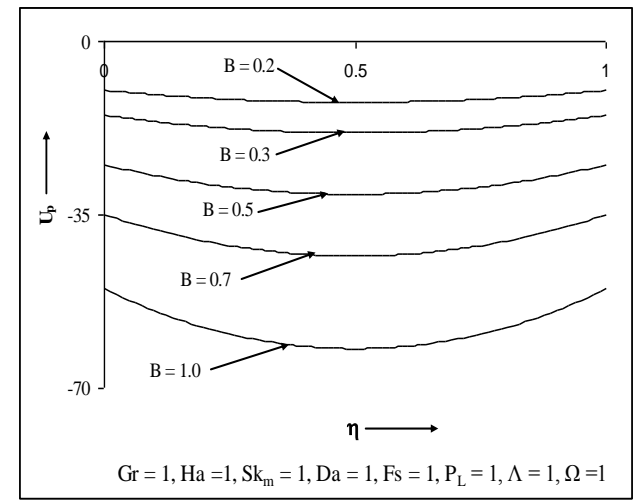

Fig. 14. $U_{p}$ versus $\eta$ for various $P_{L}$ values

The buoyancy parameter occurs as a positive body force in the fluid phase momentum equation (6) i.e. $+p_{L} B$ and therefore aids in momentum development in the fluid accounting for the boost in $\mathrm{U}$ values. Conversely the particle phase velocity, $U_{p}$, decreases substantially with a rise in $B$ value from 0.2 to 1.0 . The $U_{p}$ value for $B=0.2$, is approximately -10 ; for $\mathrm{B}=1.0$ this value falls dramatically to about -60 . In the particle phase momentum equation, the gravitational buoyancy parameter occurs as a negative body force term, $-B$ which therefore decelerates the flow with a rise in $\mathrm{B}$ values. The general results obtained for all parameter variations (with the exception of $\mathrm{Da}$ and Fs) agree well with the analytical study of Al-subaie and chamke (2003), lthough non-porous computations i.e. with $\mathrm{Da} \rightarrow$ $\infty$ and $\mathrm{Fs}=0$, were performed we have not reproduced to conserve space.

\section{Conclusions}

A mathematical model has been developed the one-dimensional free convection magnetohydrodynamic heat transfer of a particle-fluid suspension in a non-Darcian porous channel. The model has been non-dimensionalized and a number of special cases of pertinence in various engineering systems have been discussed. Finite element solutions have been obtained for both the fluid and particle phase velocity and temperature fields. Herein we have concentrated more on the velocity field computations. The present computations have shown that:

i) A rise in Grashof number (Gr) depresses fluid phase velocity, $U$, in the first half of the channel but boosts velocity in the second half of the channel.

ii) An increase in Hartmann number (Ha) decreases fluid phase velocity, $\mathrm{U}$, and also particle phase velocity, $U_{p}$, although a greater effect $s$ observed in the case of the fluid phase.

iii) A rise in inverse momentum Stokes number $\left(\mathrm{Sk}_{\mathrm{m}}\right)$ decreases fluid phase velocity, $\mathrm{U}$, but increases the particle phase velocity, $U_{p}$

iv) An increase in Darcy number (Da) strongly accelerates the flow and boosts both fluid phase velocity, $\mathrm{U}$, and the particle phase velocity, $\mathrm{U}_{\mathrm{p}}$ although the increase in the latter case is much less pronounced.

v) An increase in Forchheimer number (Fs) strongly decelerates the flow and retards flow development; both fluid phase velocity, $\mathrm{U}$, and the particle phase velocity, $U_{p}$ are both reduced. However the effect on fluid phase velocity is considerably more marked.

vi) A rise in particle loading parameter, $\mathrm{P}_{\mathrm{L}}$, has a major positive effect on fluid phase velocity, $U$, which increases considerably; the particle phase velocity, $\mathrm{U}_{\mathrm{p}}$ is also increased but relatively less so than for the fluid phase. vii) An increase in the gravitational buoyancy parameter increases the fluid phase velocity, $\mathrm{U}$, but decreases the particle phase velocity, $\mathrm{U}_{\mathrm{p}}$

The present study entails the first step in developing more sophisticated computational models of two-phase hydromagnetic flows with the objective of exploring possible refinements in existing plasma technologies. In the near future a more general transient analysis of two-phase heat transfer of a particle-suspension in an a porous medium channel will be communicated, including rotational and also anisotropic porous effects.

\section{REFERENCES}


Al-Subaie, M. and Chamkha,A., J., (2003), Analytical Solutions for Hydromagnetic Natural Convection Flow of a Particulate Suspension Through a Channel with Heat Generation or Absorption Effects, Heat and Mass Transfer J., 39, 701-707.

Bakhtar, F., Mashmoushy, H. and Jadayel, O., (1998), Modeling of two-phase flows of steam in turbines, 11th International Heat Transfer Conference, Kyongju, Korea, 23-28 August.

Bathe, K. J., (1996), Finite Element Procedures, Prentice-Hall, USA.

Bég, O. A., (1996), Non-Darcy ThermoConvection in Porous Media with MagnetoHydrodynamic and Radiative Effects, $\mathrm{PhD}$ Thesis, Reynolds Engineering Hydrodynamics Laboratory, University of Manchester, England, UK, 328 pages, March

Bég, O.A., (1998), Computational fluid dynamics modelling of dusty flows in pharmaceutical clean room environments: The Marble Generalized Model adapted for ADINA, Technical Report, Arup Manchester, UK, May.

Bég, O.A., Takhar, H.S., Kumari, M. and Nath, G.: (2001), Computational fluid dynamics modelling of buoyancy-induced viscoelastic flow in a porous medium with magnetic field effects, Int. J. Applied Mechanics and Engineering, 6, 187-210.

Bég O. A., H. S. Takhar, G. Nath and A. J. Chamkha, (2005) Mathematical and Numerical simulation of convection heat transfer in a rotating fluid in a thermallystratified high-porosity medium: Finite Difference Solutions, Int. J. Fluid Mechanics Research, May, 34, 6, 1-18 .

Bég, O. A. (2006), Transient MHD Convection of Particle-Suspensions in anisotropic porous channels: Strouhal Number Effects, In preparation for Nonlinear Analysis: Modeling and Control J., May.

Bég, O.A., Takhar, H.S., Bhargava, R., Rawat, S., (2006), Numerical simulation of biomagnetic micropolar flow in a twodimensional Darcian porous medium: a model of magnetized blood flow in tissue, Int. J. Fluid Mechanics Research, submitted, June.

Bég, O. A., Bhargava, R., Takhar, H. S., Tasveer A. Bég, S, Rawat, (2006) Computational modeling of buoyancy-induced micropolar heat and mass transfer in non-Darcy porous media with Soret/Dufour diffusion effects,
Applied Mathematical Modeling J., submitted, April.

Bhargava R., Rawat S., Takhar H.S., Beg. O.A, (2007), Pulsatile Magneto-Biofluid Flow and Mass Transfer in a Non-Darcian Porous Medium Channel, Meccanica, 42, 247-262,.

Brenner, H., Edwards, D.A. and Wasan, D.T., (1993), Interfacial Transport Processes and Rheology, Butterworth, New York, USA.

Chamkha, A., J., (1999)., Effect of Combined Particle-Phase Diffusivity and Viscosity on the Compressible Boundary Layer of a Particulate Suspension over a Flat Surface, ASME J. Heat Transfer, 121, 420-429

Chamkha, A., J., and Peddieson, J.J.R. (1991), Boundary layer flow of a particulate suspension past a flat plate, Int. J. Multiphase Flow, 17, 805-808.

Chang, H., T., Hourng, L.T., and Chien, L.,E., (1996), Application of flux-vector-splitting scheme to a dilute gas-particle JPL nozzle flow, Int. J. Numer. Methods Fluids, 22, 921-935.

Gebhart, B. et al, Buoyancy-Induced Flows and Transport, Hemisphere, Washington, USA (1988).

Greenberg, M.D., Mitra, A., and Zarroodny, S., J., (1973), A Vortex Sheet Approach to Two Phase Flows, Fourth Canadian Congress of Applied Mechanics, Montreal, Canada, May.

Hayes, W.D. and Probstein, R.F., (1966), Hypersonic Flow Theory, second edition, Academic, New York.

Hwang, C., J., and Chang, C.,G .,(1988), Numerical study of gas-particle flow in a solid rocket nozzle, AIAA J., 26, 6, 682-689.

Hossain, M.A., Das, S.K. and Pop, I., (1998), Heat transfer response of MHD free convection flow along a vertical plate to surface temperature oscillations, Int. J. NonLinear Mechanics, 33, 3, 541-553.

Ibrahim A Abbas, M F El-Amin, Amgad Salama, Effect of thermal dispersion on free convection in a fluid saturated porous medium, International Journal of Heat and Fluid Flow, 30(2) : 229-236.

Kulish, V. V., (2009), Two-phase flow through the human respiratory system, 9th Int. Symposium on Flow Visualization (2000).

Marble, F.E., (1970), Dynamics of Dusty Gases, Ann. Rev. Fluid Mechanics, 2, 397-446.

Mahdy, A.J. Chamkha,, (2010), "Chemical reaction and viscous dissipation effects on 
Darcy-Forchheimer mixed convection in a fluid saturated porous media", International Journal of Numerical Methods for Heat \& Fluid Flow, 20 (8), 924 - 940.

Moukalled, F., Darwish, M., and Sekar, B., A (2003), pressure-based algorithm for multiphase flow at all speeds, J. Computational Physics, 190, 2, $550-571$.

Mishra., S.R., Dash G., C., Acharya., M., (2012), Heat transfer in viscous free convective fluctuating MHD flow through porous media past a vertical porous plate with variable temperature., 2(6): 66-78.

Naroua, H., Ram, P.C., Sambo, A.S. and Takhar, H.S., (2000) Finite element analysis of unsteady MHD free convection flow with radiative heat transfer in a rotating fluid. J.Magnetohy -drodynamics and Plasma Research, 9, 121-140.

Osameh G., Hossein R, Mehdi A.,, (2011), "Analytical Investigation of the Effect of Viscous Dissipation on Couette Flow in a Channel Partially Filled with a Porous Medium, Transp Porous media 89(1): 1-13

Peddieson, J., (1975) Gas-particle flow past bodies with attached shock waves, AIAA J., Technical Notes, 13, 939-941.

Rao, S.S., (1982), The Finite Element Method in Engineering, Pergamon Press, Oxford.

Ram, P.C. and Takhar, H.S.: (1993), MHD free convection from an infinite vertical plate in a rotating fluid with Hall and ionslip currents, Fluid Dynamics Research, 11, 99-105.

Rawat.S, R. Bhargava, and O. Anwar Bég, (2010), Hydromagnetic micropolar free convection heat and mass transfer in a darcy forchheimer porous medium with thermophysical effects : Finite element solution, Int. J. of Appl. Math and Mech. 6 (13): 72-93,

Revankar, S.T., (1982), Free convection effects on MHD flow past a porous plate with step function change in suction velocity, Astrophys. Space Sci. J, 82, 13-23.

Sami, S. M. and Poirier, B., (1998), Two phase flow heat transfer of binary mixtures inside enhanced surface tubing, Int. Comm. Heat Mass Transfer , 25, 6, 763-775.

Singh, K. R. and Cowling, T.J., (1963), Thermal convection in magneto-hydrodynamics, $Q . J$. Mech. Appl. Math, 16, 1-15.

Satyamurthy, P., Thiyagarajan, T., K., N. S. Dixit, Venkatramani N., and Mushtaq, A., (1997), Two-phase flow studies of mercurynitrogen in simulated liquid metal magnetohydrodynamic energy convertors of gravity type, J. Magnetohydrodynamics, 33, 4, 431-437.

Soundalgekar, V.M. and Takhar, H.S., (1977), On MHD and heat transfer over a semi-infinite plate under a transverse magnetic field, Nuclear Engineering and Design, 42, 233236.

Surma Devi, C.D., Takhar. H.S. and Nath, G.: (1986), MHD flow past a cone, Acta Technica Csav, 31, 400-409.

Takhar, H.S. and Soundalgekar, V.M., Dissipation effects on MHD free convection flow past a semi-infinite vertical plate, Applied Scientific Research, 36, 163-171.

Takhar. H.S. and Pop, I., (1984) On MHD heat transfer from a wedge at large Prandtl numbers, Mechanics Research Communications, 11, 191-194.

Takhar, H.S., Agarwal, R.S., Bhargava, R. and Jain, S., (1998), Mixed convective nonsteady 3-dimensional micropolar fluid flow at a stagnation point, Heat and Mass Transfer J., 33, 443-448.

Takhar, H.S., Bhargava, R. and Agarwal, R.S.: (2001), Finite element solution of a micropolar fluid from an enclosed rotating disk with suction and injection. Int. J. Eng. Sci., 39, 913-927.

Thacker, W.T., Watson, L.T. and Kumar, S.K., (1988), MHD free convection from a disk rotating in a vertical plane, Appl. Math. Modelling, 14, 527-537.

Usha, R.,Senthilkumar, S., and Tulapurkara, E. G. , (2005), Numerical study of particulate suspension flow through wavy-walled channels, Int. J. Numerical Methods in Fluids.

Wilks, G., (1976), MHD free convection about a semi-(1980)Appl. Math. Phys (ZAMP), 27, 621-631.

W. H. Leung, S. T. Revankar, Y. Ishii and M. Ishii, (1993), Interfacial area and two-phase flow structure development in bubbly flow measurement by double probe sensor, American Nuclear Society Proc., 1993 National Heat Transfer Conf., Atlanta, USA, Volume 7, 51-58. 